\title{
Protocol for a randomised trial of early kangaroo mother care compared to standard care on survival of pre-stabilised preterm neonates in The Gambia (eKMC)
}

Helen Brotherton ${ }^{1,2,3^{*}}$ (D), Abdou Gai ${ }^{2}$, Cally J. Tann ${ }^{1,4,5}$, Ahmadou Lamin Samateh ${ }^{6}$, Anna C. Seale ${ }^{1}$, Syed M. A. Zaman', Simon Cousens ${ }^{1}$, Anna Roca ${ }^{2}$ and Joy E. Lawn ${ }^{1}$

\begin{abstract}
Background: Complications of preterm birth cause more than 1 million deaths each year, mostly within the first day after birth (47\%) and before full post-natal stabilisation. Kangaroo mother care (KMC), provided as continuous skin-to-skin contact for $18 \mathrm{~h}$ per day to fully stabilised neonates $\leq 2000 \mathrm{~g}$, reduces mortality by $36-51 \%$ at discharge or term-corrected age compared with incubator care. The mortality effect of starting continuous KMC before stabilisation is a priority evidence gap, which we aim to investigate in the eKMC trial, with a secondary aim of understanding mechanisms, particularly for infection prevention.
\end{abstract}

Methods: We will conduct a single-site, non-blinded, individually randomised, controlled trial comparing two parallel groups to either early (within $24 \mathrm{~h}$ of admission) continuous KMC or standard care on incubator or radiant heater with KMC when clinically stable at $>24 \mathrm{~h}$ of admission. Eligible neonates $(n=392)$ are hospitalised singletons or twins < $2000 \mathrm{~g}$ and $1-24 \mathrm{~h}$ old at screening who are mild to moderately unstable as per a trial definition using cardiorespiratory parameters. Randomisation is stratified by weight category $(<1200 \mathrm{~g} ; \geq 1200 \mathrm{~g})$ and in random permuted blocks of varying sizes with allocation of twins to the same arm. Participants are followed up to $28 \pm 5$ days of age with regular inpatient assessments plus criteria-led review in the event of clinical deterioration. The primary outcome is all-cause neonatal mortality by age 28 days. Secondary outcomes include the time to death, cardio-respiratory stability, hypothermia, exclusive breastfeeding at discharge, weight gain at age 28 days, clinically suspected infection (age 3 to 28 days), intestinal carriage of extended-spectrum beta-lactamase producing (ESBL) Klebsiella pneumoniae (age 28 days), and duration of the hospital stay. Intention-to-treat analysis will be applied for all outcomes, adjusting for twin gestation.

Discussion: This is one of the first clinical trials to examine the KMC mortality effect in a pre-stabilised preterm population. Our findings will contribute to the global evidence base in addition to providing insights into the infection prevention mechanisms and safety of using this established intervention for the most vulnerable neonatal population.

Trial registration: ClinicalTrials.gov NCT03555981. Submitted 8 May 2018 and registered 14 June 2018. Prospectively registered.

Keywords: Preterm, Neonate, Kangaroo care, Kangaroo mother care, Skin-to-skin contact, Survival, Infection, Randomised controlled trial, Pragmatic

\footnotetext{
* Correspondence: helen.brotherton@lshtm.ac.uk; hbrotherton@mrc.gm

${ }^{1}$ Faculty of Epidemiology and Population Health, and MARCH Centre,

London School of Hygiene \& Tropical Medicine (LSHTM), Keppel Street,

London, UK

${ }^{2}$ MRC Unit The Gambia at LSHTM, Atlantic Road, Fajara, The Gambia

Full list of author information is available at the end of the article
}

(c) The Author(s). 2020 Open Access This article is distributed under the terms of the Creative Commons Attribution 4.0 International License (http://creativecommons.org/licenses/by/4.0/), which permits unrestricted use, distribution, and reproduction in any medium, provided you give appropriate credit to the original author(s) and the source, provide a link to the Creative Commons license, and indicate if changes were made. The Creative Commons Public Domain Dedication waiver (http://creativecommons.org/publicdomain/zero/1.0/) applies to the data made available in this article, unless otherwise stated. 


\section{Background}

Every year an estimated 14.8 million neonates are born preterm (<37 completed weeks of gestation), of which $>$ $80 \%$ are in Asia or Sub-Saharan Africa [1], and more than 1 million die due to complications of prematurity [2]. An estimated $47 \%$ of all prematurity-related deaths in resource-limited settings occur within the first day after birth [3] before post-natal stabilisation is complete. This is the critical period in which to target interventions to improve preterm survival and accelerate progress toward the Sustainable Development Goal (SDG) target 3.2 for neonatal mortality reduction. More than 40 countries, many in sub-Saharan Africa, need to more than double their current progress to meet the target by 2030 [4].

Kangaroo mother care (KMC) is an evidence-based package recommended as standard care for all clinically stable (pre-stabilised) neonates $<2000 \mathrm{~g}$ [5], which is the proxy weight used in previous KMC trials as an indicator for preterm birth [6]. Described in Colombia four decades ago, KMC has since been widely adopted as a cornerstone of neonatal care. The key component is prolonged, skin-to-skin contact between neonate and caregiver, facilitating exclusive breastmilk feeding and shorter hospital stay [7].

Clinical stability is variably defined in previous KMC trials with no standardised WHO definition or validated clinical model for resource-limited settings. In neonates $<2000 \mathrm{~g}$ who have completed stabilisation or post-natal transition, continuous KMC (aiming for $>18 \mathrm{~h} /$ day) reduces mortality at discharge or 40 weeks post-menstrual age by $36-51 \%[6,8,9]$ compared to incubator care, with the mortality effect observed only in resourcelimited settings [6]. However, an evidence gap exists for neonates yet to complete stabilisation, who have greatest risk of death or adverse outcome [6]. In 20 trials that assessed mortality at latest follow-up and were included in three systematic reviews $[6,8,9], \mathrm{KMC}$ was initiated at an average age $\leq 4$ days in seven trials, with only one RCT starting continuous KMC in pre-stabilised neonates within $24 \mathrm{~h}$ after birth [10]. This Ethiopian trial reported a $40 \%$ reduction in mortality ( $R R=0.57,95 \%$ CI $0.33-$ $1.00, p<0.05)$ but more than half of the unstable neonates were excluded, and the eligibility criteria were unclear, leading to high risk of bias $[6,10]$.

$\mathrm{KMC}$ is a safe intervention for unstable neonates in resource-rich settings with intensive monitoring [11], but the safety profile in a context of less close clinical monitoring is not established [6] and warrants further scrutiny.

KMC works through multiple pathways, many mediated by skin-to-skin contact [12], including thermal control [6], neuro-endocrine mechanisms involving oxytocin release in both mother and neonate [12], reduced cortisol and stress response [13], cardio-respiratory stabilisation [14], enhanced breast milk production [6] and empowerment of the KMC provider in caring for their baby. Alterations in the neonatal microbiome with intermittent KMC have also been reported [15] and warrant further exploration to understand the infection prevention effects of KMC. The relevance and relative contribution of these mechanisms for KMC in pre-stabilised neonates are unknown, particularly for infection prevention outcomes, which is an evidence gap for all preterm neonates.

The eKMC trial aims to investigate continuous KMC in pre-stabilised neonates $<2000 \mathrm{~g}$ in a Gambian health facility setting. A secondary aim is to explore potential underlying mechanisms of $\mathrm{KMC}$ in this high-risk population.

\section{Objectives}

The primary objective of the eKMC trial is to assess the effect of early continuous KMC on the survival of prestabilised preterm neonates.

\section{Secondary objectives}

Secondary objectives include the following:

1. Assess the effect of early continuous KMC on other important clinical outcomes (growth, late-onset infections and duration of hospital stay)

2. Evaluate the safety of providing early continuous KMC to pre-stabilised preterm neonates in a resource limited facility setting

3. Explore possible mechanisms for hypothesised beneficial effects of early continuous KMC in prestabilised preterm neonates, focusing on infection prevention

\section{Methods/design}

This article has been prepared according to the Standard Protocol Items: Recommendations for Interventional Trials (SPIRIT) statement (Additional file 1) [16].

\section{Study design}

This single-site, pragmatic, non-blinded, individually randomised superiority trial compares two parallel groups managed with either continuous KMC started within $24 \mathrm{~h}$ of hospital admission or standard care with intermittent or continuous KMC when clinically stable $>24 \mathrm{~h}$ after admission. The unit of randomisation is the mother in a 1:1 ratio with twin participants randomised to the same arm.

\section{Study setting and context}

Recruitment began on 23 May 2018 and is ongoing at the neonatal unit of Edward Francis Small Teaching Hospital $(E F S T H)$, the main neonatal referral unit in The Gambia, with research support from the MRC Unit 
of Gambia at London School of Hygiene \& Tropical Medicine (MRCG at LSHTM).

The Gambia is the smallest country in mainland Africa, with a population of 2.1 million, and it is ranked 174/189 on the Human Development Index (2017) [17]. Neonatal mortality declined from 49 to 26 per 1000 live births between 1990 and 2018, respectively [18], with $12-14 \%$ of Gambian neonates born preterm $[1,19]$ and $29 \%$ of neonatal deaths attributed to complications of prematurity [3].

A quarter (26\%) of the 1400 annual neonatal admissions to EFSTH are due to prematurity [20], and the neonatal case fatality rate is $38 \%$, with the highest rate (58\%) occurring amongst neonates born $<1500 \mathrm{~g}$ [20]. Both in-born (born at the EFSTH maternity unit) and out-born (born at another health facility or home) neonates are admitted from a mixed rural/urban population.

Neonatal care is typical of secondary level "neonatal special care" [21] and includes management in incubators or under radiant heaters, respiratory support via oxygen concentrators or continuous positive airway pressure (bubble-CPAP), phototherapy, feeding support via gastric tubes and intravenous (IV) fluids, caffeine or aminophylline, phenobarbitone and broadspectrum antibiotics. Invasive ventilation, surfactant, IV fluid pumps and continuous cardio-respiratory monitoring are unavailable. Continuous KMC was implemented as standard care during the formative trial phase in September 2017. Intermittent KMC is provided for a minimum of $60 \mathrm{~min}$ at periodic intervals on the neonatal unit once the neonate is off respiratory support and establishing enteral feeds. Neonates $<2000 \mathrm{~g}$ receive continuous KMC on an adjacent eight-bed KMC unit once they are stable in room air, are tolerating full enteral feeds and have a willing caregiver available. Neonates are transferred to the KMC unit at average 10 days of age $(n=148$, SD 7.8) with the average KMC unit admission lasting for 6.9 days $(n=108$, SD 4.0$)$ and $92 \%(141 / 151)$ of discharged neonates attend hospital follow-up at least once, on average 7.5 days after discharge $(n=141$, range 2-23 days) (unpublished audit data, Sept. 2017 to May 2018, H. Brotherton).

\section{Study population and procedures}

Enrolment, interventions and assessments are outlined in Fig. 1.

The study population is hospitalised neonates $<2000 \mathrm{~g}$ and age $1-24 \mathrm{~h}$ old at the start of the screening who meet the trial definition of mild-moderate instability based on cardio-respiratory parameters and respiratory support provision (Fig. 2).

Inclusion criteria are as follows:
- New admission of singleton or twin (inborn or outborn)

- Weight $<2000 \mathrm{~g}$ as per study scale

- Age 1-24h old when screening begins

- Mother or other caregiver available and willing to provide intervention

Exclusion criteria are as follows:

- Triplets who are all admitted to the study site

- Congenital malformation not compatible with life or needing immediate surgical intervention

- Severe jaundice

- Seizures

- Stable as assessed during cardio-respiratory screening

- Severely unstable as assessed during cardiorespiratory screening or died during screening

- No study bed available

- Neonates/mothers enrolled in another research study

- No written informed consent from parent or caregiver within $24 \mathrm{~h}$ of admission.

\section{Screening for eligibility}

Eligibility is assessed in all admitted neonates with referral weight $\leq 2000 \mathrm{~g}$ as soon as possible and once > $1 \mathrm{~h}$ old. Weight is confirmed using a calibrated SECA $^{\mathrm{Tx}} 757$ digital weighing scale, and source documents are checked for age and other study involvement. All potentially eligible neonates aged $<24 \mathrm{~h}$ undergo an examination with cardio-respiratory stability assessed over 10 min using $\operatorname{Nonin}^{\text {тм }}$ 2500A pulse oximeter.

Stable neonates are excluded as it is considered unethical to randomise them to a proven intervention. Mildly unstable neonates are immediately eligible for recruitment. Moderately or severely unstable neonates undergo continuous pulse oximetry with a repeat stability assessment $3 \mathrm{~h}$ later. Severely unstable neonates are excluded at the repeat $3 \mathrm{~h}$ screening, as it is not possible to provide KMC alongside resuscitation or CPAP at the study site (Fig. 2). Clinically eligible neonates are recruited if a study bed is available and a caregiver is willing to both provide the intervention (if applicable) and give written consent within $24 \mathrm{~h}$ of hospital admission. If eligibility criteria are met but the caregiver is only available $>3 \mathrm{~h}$ after the end of cardio-respiratory screening, stability is re-checked prior to consenting to avoid inadvertent recruitment of stable or severely unstable patients. Standard care under radiant heater or incubator is provided to all neonates during the screening period. 


\begin{tabular}{|c|c|c|c|c|c|c|c|c|c|c|c|c|c|c|}
\hline \multirow[b]{2}{*}{ Timeline } & \multirow{2}{*}{$\begin{array}{l}\text { Enrolment } \\
<24 \mathrm{~h} \text { of } \\
\text { admission }\end{array}$} & \multirow{2}{*}{$\begin{array}{l}\text { Baseline } \\
\text { ass'ment }\end{array}$} & \multirow[t]{2}{*}{ Allocation } & \multicolumn{10}{|c|}{ Post-Allocation } & \multirow{2}{*}{$\begin{array}{l}\begin{array}{l}\text { Close } \\
\text { out }\end{array} \\
\text { D28+/- } \\
5 \text { d }\end{array}$} \\
\hline & & & & $\mathrm{TO}^{1}$ & T6 & T12 & T18 & T24 & D2 & $\mathrm{D}^{2}$ & D7 & D14 & D21 & \\
\hline ENROLMENT: & \multirow{4}{*}{\multicolumn{14}{|c|}{$\begin{array}{l}x \\
X\end{array}$}} \\
\hline Eligibility screening ${ }^{3}$ & & & & & & & & & & & & & & \\
\hline Informed consent & & & & & & & & & & & & & & \\
\hline Allocation & & & & & & & & & & & & & & \\
\hline \multirow{3}{*}{\multicolumn{15}{|c|}{$\begin{array}{l}\text { INTERVENTIONS: } \\
\text { Control } \\
\text { Intervention }\end{array}$}} \\
\hline & & & & & & & & & & & & & & \\
\hline & & & & & & & & & & & & & & \\
\hline \multicolumn{15}{|l|}{ ASSESSMENTS: } \\
\hline Temperature & & $x$ & & $x$ & $x$ & $x$ & $x$ & $x$ & $\mathrm{x}$ & $x$ & $\mathrm{x}$ & $x$ & $x$ & \\
\hline Glucose & & $x$ & & $x$ & $x$ & $x$ & $x$ & $x$ & $x$ & $x$ & $x$ & $x$ & $x$ & \\
\hline Stability $^{3}$ & & $x$ & & $x$ & $x$ & $x$ & $x$ & $x$ & $x$ & $x$ & $x$ & $x$ & $x$ & \\
\hline Examination & & $x$ & & $\longleftarrow$ & & & & $\longrightarrow$ & & & & & & \\
\hline \multicolumn{15}{|l|}{ Gestational age } \\
\hline Weight & & $x$ & & & & & & & & $x^{4}$ & $x$ & $x$ & $x$ & $x$ \\
\hline Length & & & & & & & & & & & $x$ & $x$ & $x$ & $x$ \\
\hline Head circumference & & $x$ & & & & & & & & & $x$ & $x$ & $x$ & $x$ \\
\hline Neonatal rectal swab & & $x$ & & & & & & & & & $x$ & & & $x$ \\
\hline Neonatal skin swab & & $x$ & & & & & & & & & $x$ & & & $x$ \\
\hline $\begin{array}{l}\text { KMC provider skin } \\
\text { swab }^{5}\end{array}$ & & $x$ & & & & & & & & & & & & \\
\hline $\begin{array}{l}\text { Maternal recto-vaginal } \\
\text { swab }\end{array}$ & & $x$ & & & & & & & & & & & & \\
\hline \multicolumn{15}{|l|}{ Outcome variables } \\
\hline 1.All-cause mortality & & & & & & & & & & & & & & $x$ \\
\hline 2.Time to death & & & & & & & & & & & & & & \\
\hline 3.Stability (aSCRIP) & & & & & & & & $x$ & & & & & & \\
\hline 4. Temperature $<36.5^{\circ} \mathrm{C}$ & & & & & & & & $x$ & & & & & & \\
\hline 5. Weight gain & & & & & & & & & & & & & & $x$ \\
\hline 6.Exclusive & & & & & & & & & & & & & & \\
\hline $\begin{array}{l}\text { breastfeeding at } \\
\text { discharge }^{6}\end{array}$ & & & & & & & & & & & & & & \\
\hline 7.Suspected late onset & & & & & & & & & & & & & & \\
\hline infection & & & & & & & & & & & & & & \\
\hline $\begin{array}{l}\text { 8. Intestinal ESBL } K \\
\text { pneumoniae carriage }\end{array}$ & & & & & & & & & & & & & & $x$ \\
\hline 9.Duration of stay ${ }^{6}$ & & & & & & & & & & & & & & \\
\hline
\end{tabular}

Fig. 1 eKMC trial schedule of enrolment, interventions and assessments [16] 1. The start of study procedures (Time 0) is defined as when the pulse oximeter is attached for baseline continuous cardio-respiratory assessment, immediately prior to the intervention/control procedures commencing. 2. Participants are reviewed daily until KMC unit admission, after which they are reviewed on days 7, 14, 21, and 28 of age whilst inpatients and on day 28 as outpatients. Daily reviews are re-started if the baby is transferred back to the neonatal unit. 3. Stability definitions used during eligibility screening and routine assessments are detailed in Fig. 2. 4. Weight at 5 days of age is taken on calibrated digital scales and then is taken daily until either discharge or KMC unit admission, after which it is obtained on days 7, 14, 21, and 28 whilst an in-patient and at the day 28 follow-up if discharged. 5. Skin swab samples are taken from the first person to provide skin-to-skin contact and the mother (if different) as soon as possible and prior to any skin-to-skin contact. The relationship of the KMC provider to the participant is documented and correlated with swabs using unique, anonymised identification codes. 6 . Outcomes such as feeding method and duration of stay are recorded at the time of discharge, including for participants hospitalised for $>28$ days

\section{Consent}

Sensitisation activities with health workers, pregnant women and families are conducted at referral health facilities to support recruitment. Written, informed consent for participation and provision of continuous KMC (in event of randomisation to intervention arm) is sought from the first available caregiver at the study site within
$24 \mathrm{~h}$ of admission by trained study personnel. The parent is the preferred person to provide informed consent, but other relatives may provide consent with parental informed consent being sought as soon as possible. Consent is requested in English with verbal translation into local languages using a pre-designated dictionary of definitions. Impartial witnesses are used to support the consenting 


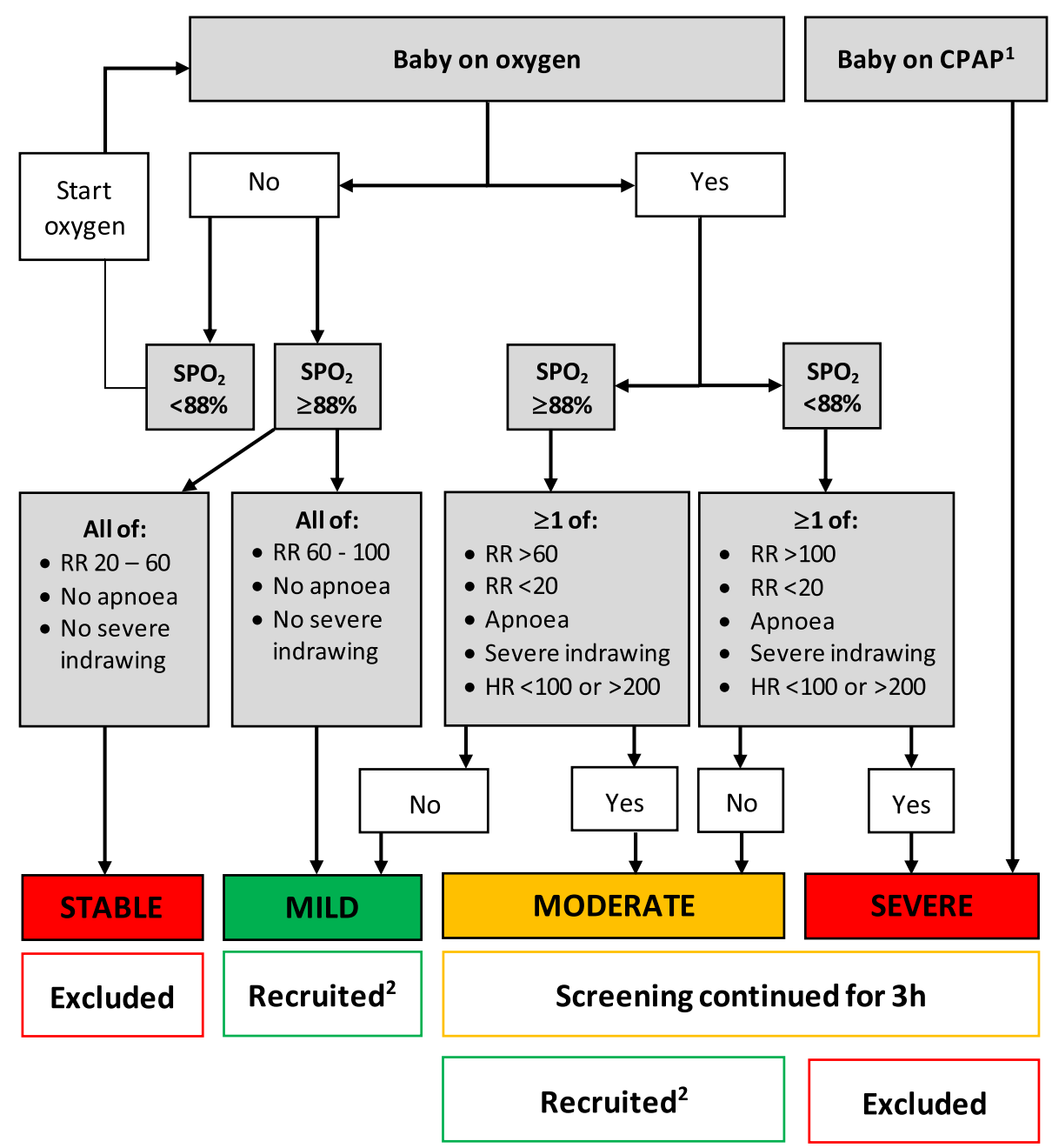

\section{CLINICALLY ELIGIBLE}

$\mathrm{CPAP}=$ Continuous positive airway pressure, provided as bubble CPAP; RR=Respiratory rate; $\mathrm{h}=$ hours; $\mathrm{HR}=$ Heart rate; $\mathrm{SPO}_{2}=$ oxygen saturation

Fig. 2 eKMC trial definitions of cardio-respiratory instability and eligibility status. 1. Criteria for starting CPAP is a Silverman-Anderson score $\geq 4$ that does not improve with oxygen therapy and the absence of the following: heart rate $<100 \mathrm{bpm}$, floppy tone and seizures. 2 . The neonate is recruited if a study bed is available and consent is provided by a willing caregiver

process with caregivers who are unable to read or write English. Consent for obtaining and future use of paired maternal recto-vaginal and skin swab samples from the first KMC provider and mother (if different) is sought before any skin-to-skin contact occurs.

\section{Randomisation, allocation and blinding}

An independent statistician generated a randomisation sequence using VBA (Visual Basic Application) within an Access database to produce two random number tables with stratification by admission weight categories $(<1200$ $\mathrm{g}$ or $\geq 1200 \mathrm{~g}$ ). Random permuted blocks of varying block sizes were used in a 1:1 allocation. The allocation sequence is concealed with sequentially numbered, opaque, sealed envelopes prepared by an independent researcher and accessible to study team only. Following the collection of baseline data, the study nurse opens the next numbered envelope for the correct weight category. The participant identifier, date and time are recorded on the outside of the envelope prior to opening, to identify any subversion of allocation sequence. Twins are allocated to the same arm, according to the first eligible twins' weight.

Given the nature of KMC, blinding parents/caregivers and study personnel to the allocation arm and the 
primary outcome is not possible. Process and secondary outcome data will be anonymised, and all analyses will be blinded.

\section{Intervention}

The terms KMC and skin-to-skin contact are used as synonyms in the literature, but the intervention under study is continuous skin-to-skin contact between neonate and caregiver started within $24 \mathrm{~h}$ of admission. The neonate is naked except for nappy and woollen hat and is secured with a Thari wrapper (customised KMC wrapper developed in South Africa) in a prone, frog-leg position on caregivers' naked chest with head turned sideways (Fig. 3).

The caregiver sits or lies down whilst the neonate receives all other treatments (oxygen via nasal prongs, intravenous (IV) fluids via peripheral venous cannula, gastric tube feeds and IV medications). If the mother is unavailable, other relatives (e.g., fathers or grandmothers) provide the intervention. KMC is advised for as long as possible, aiming for $\geq 18 \mathrm{~h} /$ day. When not receiving $\mathrm{KMC}$, the baby remains in an incubator or under a radiant heater in the same room, with co-habitation of the radiant heater. If participants meet clinical "stopping criteria” (Fig. 4c), participants are temporarily withdrawn from the intervention arm, receive standard incubator

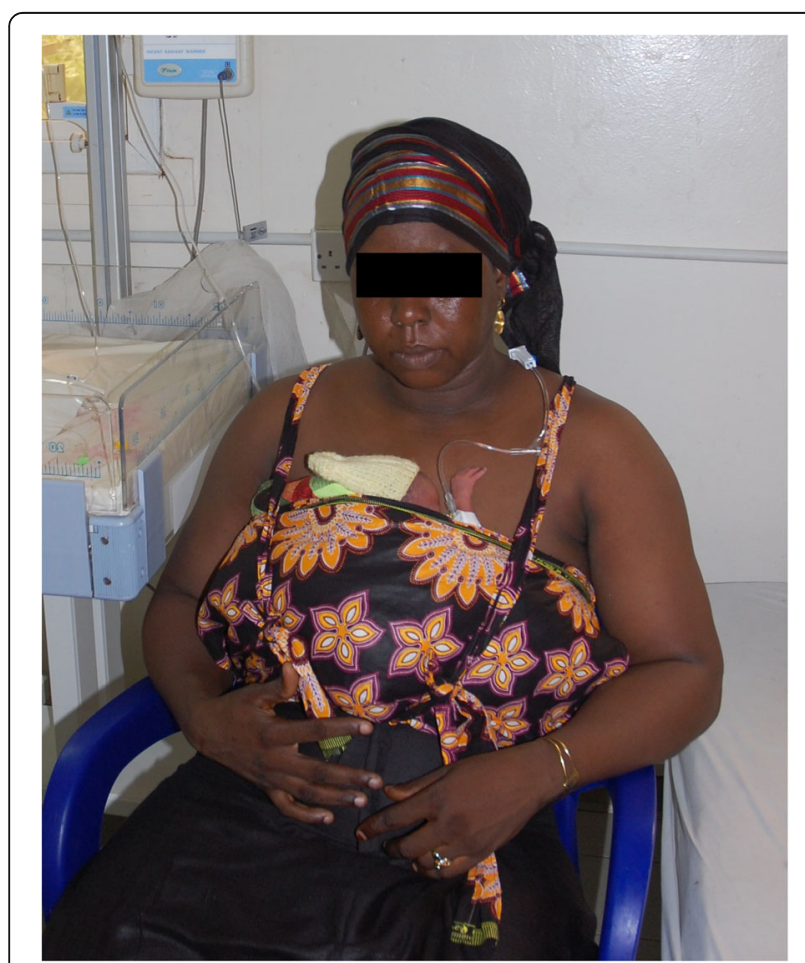

Fig. 3 An eKMC participant receiving the intervention of ocntinuous skin-to-skin contact at the same time as other standard care treatments (H.Brotherton with caregiver consent for publication) or radiant heater care and re-start KMC once the stability criteria are met (Fig. $4 \mathrm{~d}$ )

\section{Control}

The neonate is managed in an incubator or under a radiant heater, naked except for a woollen hat and nappy or wrapped in a cloth. The parent/caregiver can touch, hold and feed the neonate as per standard practice but skinto-skin contact is not provided until stability criteria are met (Fig. 4d) and after $>24 \mathrm{~h}$ since hospital admission. Participants then receive intermittent $\mathrm{KMC}$ on the neonatal unit and continuous $\mathrm{KMC}$ on the adjacent $\mathrm{KMC}$ unit (Fig. 4d).

\section{Flow around study site for both arms}

After their baseline stability data have been collected, all participants are transferred to a "trial area" within the neonatal unit containing four small beds, chairs, incubators, radiant heater and an oxygen concentrator. This area can accommodate 8-10 patients with twin participants sharing incubators. If a neonate subsequently becomes severely unstable (Fig. 2), the affected participants are transferred to the high dependency area and then follow the standard flow around the neonatal unit. Neonates are moved from "trial area" to the KMC unit once stability criteria are met (Fig. 2), full enteral feeds have been tolerated for the previous $12 \mathrm{~h}$, no phototherapy is required and both a willing caregiver and $\mathrm{KMC}$ unit bed are available. If participants become unwell whilst on the KMC unit, they are re-admitted to the neonatal unit and follow the standard patient flow.

\section{Clinical management and study procedures for neonates in both arms}

Baseline anthropometric and clinical data are collected prior to randomisation with the exception of gestational age and length (within $48 \mathrm{~h}$ of recruitment) and sociodemographic data (within 28 days). The first available caregiver is sensitised at baseline for infection control, provision of $\mathrm{KMC}$, clinical danger signs and when to call for help. All other routine and emergency treatments, including discharge, are provided according to a standardised preterm management protocol, based on preexisting standard care at the study site and consistent with WHO guidelines. Compliance with the protocol is monitored prospectively by trial clinicians. Continuous monitoring of cardio-respiratory stability with a Nonin ${ }^{\text {тм }}$ $2500 \mathrm{~A}$ pulse oximeter occurs for a minimum $24 \mathrm{~h}$ of study participation, until stability is reached (Fig. 2). Direct nursing observation documents all details of the KMC provided, including the date and time of first KMC contact, relationship with the person providing $\mathrm{KMC}, \mathrm{KMC}$ session frequency and duration, number of 


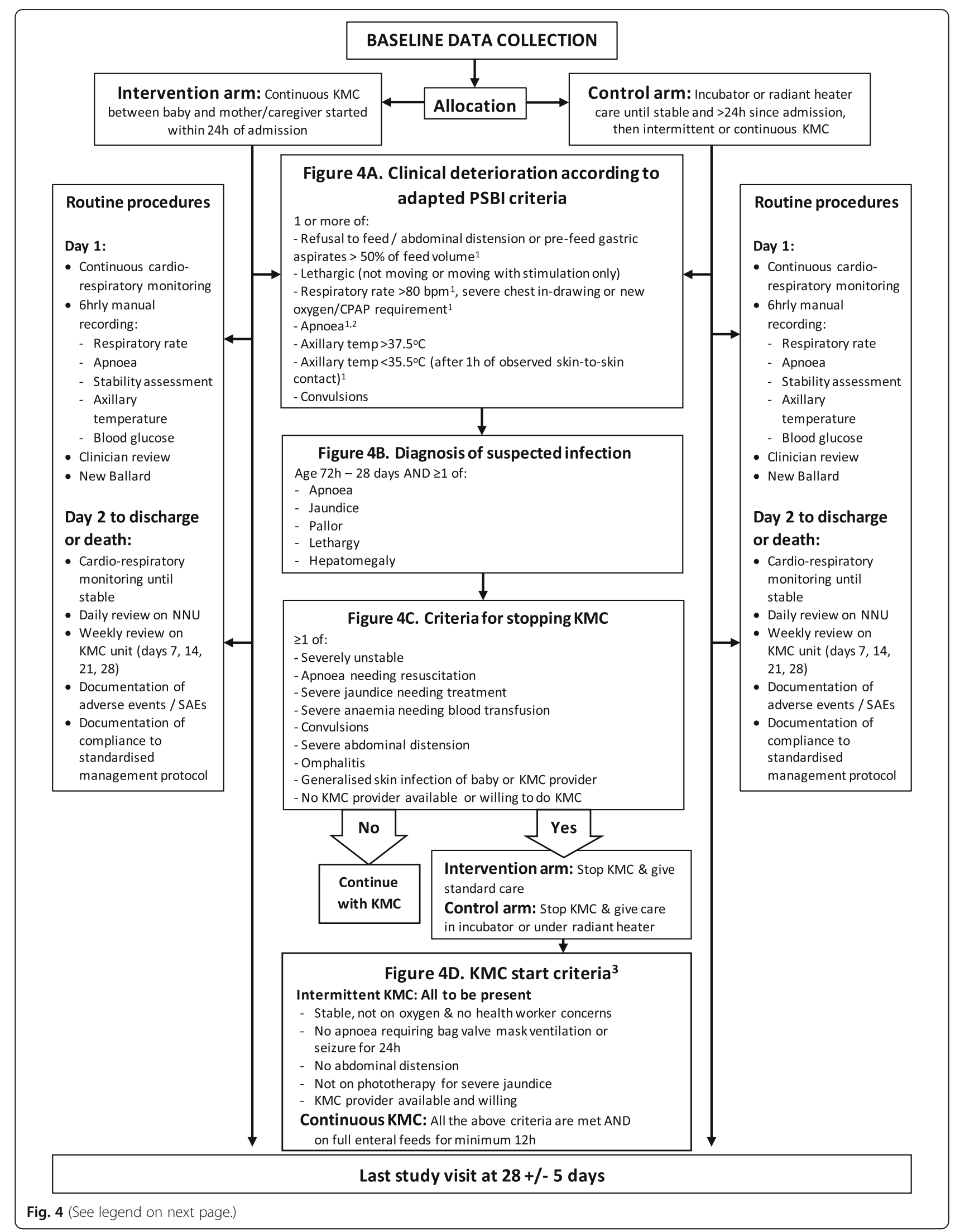


(See figure on previous page.)

Fig. 4 Overview of eKMC routine procedures and assessment of clinical deterioration including key trial criteria. 1. New or changed PSBI

definitions to increase relevance for hospitalised preterm neonates. 2. Spontaneous apnoea with no identifiable reason, e.g., not associated with

milk aspiration or end-stage respiratory failure. 3. Re-start criteria also apply to neonates in control arm at the initiation of KMC

neonates receiving $\mathrm{KMC}$ from the same provider and the reason for not providing KMC.

Structured study reviews occur with decreasing intensity as stability improves, with reviews every $6 \mathrm{~h}$ for the first $24 \mathrm{~h}$, daily reviews whilst on the neonatal unit and weekly reviews during the KMC unit admission (Fig. 4). The final study review at $28 \pm 5$ days of age occurs at EFSTH, with home visits for non-attenders. Caregivers may withdraw from the study at any time. Data collected up to the point of the most recent follow-up within $28 \pm$ 5 days of age will be included in the analyses.

\section{Outcome measures}

Primary outcome

The primary outcome is all-cause mortality at age 28 days.

\section{Secondary outcomes}

Secondary outcomes include the following:

1. Time from start of study procedures to death The date and time of death is recorded as soon as possible using the death certificate as a source document for in-hospital deaths and according to the caregiver verbal report for out-of-hospital deaths.

2. Cardio-respiratory stability at $\mathbf{2 4} \mathrm{h}$ of study participation (aSCRIP score)

The Stability of Cardio-Respiratory in Preterm Infants (SCRIP) score is an objective measure of stability used in previous KMC trials [14, 22]. The score was modified for relevance to a pre-stabilised preterm population receiving oxygen (Additional file 2).

3. Prevalence of hypothermia (axillary temperature < $36.5^{\circ} \mathrm{C}$ ) at $24 \mathrm{~h}$ of study participation

Axillary temperature is measured with an electronic thermometer as the average of three consecutive values.

4. Proportion of neonates exclusively breastfeeding at the time of discharge Exclusive breast-feeding and use of formula milk are recorded prospectively by direct observation and questioning of caregiver at time of discharge.

5. Mean daily weight gain at age $28 \pm \mathbf{5}$ days (g/day) This gain is the difference in weight between baseline and day $28 \pm 5$ days, as measured on a calibrated study scale.

6. Incidence of clinically suspected infection from 3 to 28 days of age or latest follow-up
In the absence of a standardised clinical definition for infection in preterm neonates, a two-step process is used to identify clinically suspected infection (Fig. 4a \& b). The WHO's Possible Serious Bacterial Infection (PSBI) criteria [23] were adapted to increase the relevance to a hospitalised preterm population receiving KMC (Fig. 4a). If any aPSBI criteria are present, a clinician examines the baby for features of suspected infection [24] (Fig. 4b), and blood \pm cerebro-spinal fluid (CSF) cultures are obtained if these criteria are met. BACTEC Peds $\mathrm{Plus}^{\mathrm{nt}} / \mathrm{F}$ vials are inoculated with minimum $1 \mathrm{ml}$ venous blood by study clinicians and processed as soon as possible within $24 \mathrm{~h}$ in an automated Bactec 9050 BD machine at MRCG at LSHTM. Samples with positive signal undergo sub-culture as per standard culture methods, species identification by API 80 system and antibiotic susceptibility testing by disc diffusion according to CLSI 2017 guidelines. CSF samples are collected by study clinicians as soon as possible and in the absence of contraindications. CSF is transported to MRCG laboratories at room temperature within $1 \mathrm{~h}$ of collection for routine microbiological and biochemical analysis. Isolation of clinically significant bacteria are recorded, with coagulase negative staph (CONS) and bacillus species predefined as non-pathogenic. A secondary analysis of the effect of KMC on confirmed (culture positive) infection is planned.

7. Prevalence of neonatal intestinal carriage of extended-spectrum beta-lactamase (ESBL)-producing Klebsiella pneumoniae at age $28 \pm 5$ days Rectal swabs are taken with size appropriate $\mathrm{FLOQ}^{\mathrm{m}}$ swabs and stored for batch microbiological processing. Additional paired maternal and/ or caregiver-neonatal carriage flocked swab samples obtained at baseline, 7 days (neonatal) and $28 \pm 5$ days (neonatal) (Fig. 1) are stored for future microbiological and molecular processing.

\section{Mean duration of stay (hours)}

Time from study site admission to discharge is documented prospectively according to source documents for the first admission episode. This information indicates if a participant is discharged after 28 days of age.

\section{Other variables of interest}

Adverse events (e.g., abnormal blood glucose, jaundice, apnoea) are observed in both arms as safety parameters. 
The number, proportion and reason for temporary withdrawal from the intervention arm is recorded. Weekly anthropometry (weight, length and head circumference) provides additional indicators of growth. Continuous heart rate and oxygen saturation measurements alongside 6-hourly aSCRIP scores (Additional file 2) are recorded for the first $24 \mathrm{~h}$ of study participation for a planned secondary analysis of cardio-respiratory stability.

\section{Data collection, management and security}

All study personnel are trained in ICH-GCP, study objectives and study-specific procedures, in addition to being trained in clinical newborn care and KMC. Sociodemographic, clinical and summary laboratory data are collected using the REDCap ${ }^{\text {Tw }}$ data entry system with built-in range and consistency checks. Length is obtained with a Seca210 measuring mat and head circumference with non-stretchable tape measures using triplicate measures and regular inter- and intra-observer standardisation checks with double-blind assessments against clinician assessment. Vital signs are measured over 10-min periods to generate mean values, using calibrated Nonin $^{\text {tw }}$ 2500A pulse oximeters for heart rate and oxygen saturation with manual recording of respiratory rate. Gestational age assessment is done by trained clinicians using the New Ballard [25] score with regular inter-observer variability monitoring. All biological samples are processed or stored (maximum $-70^{\circ} \mathrm{C}$ ) at MRCG at LSHTM laboratories and biobank (ISO 15189 Accredited), including paired neonatal-caregiver carriage swab samples and invasive isolates intended for future exploration of infection mechanisms. Cardio-respiratory stability data from Nonin $^{\text {TM }}$ 2500A pulse oximeters is

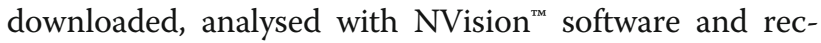
onciled with the study database. The daily dose of KMC is automatically calculated before reconciliation with the study database. All data are securely stored on a MRCG central server or at the study site with restricted access. A non-identifiable unique study number for neonate and caregiver is used to maintain confidentiality for all data, including stored samples, with linkage of neonatal and caregiver identifications.

\section{Sample size}

A total of 392 subjects (1:1 ratio) is required to detect a $30 \%$ relative reduction in the primary outcome (power $80 \%$, alpha $=0.05)$ with recruitment planned for 2 years. This number is based on an expected mortality rate of $48 \%$ [20], with adjustment for an estimated 15\% reduction in mortality due to trial implementation. Loss to follow-up rates are expected to be low $(<3 \%)$ due to the restricted geographical area, co-ordination of follow-up with routine appointments and re-imbursement of travel expenses.

\section{Statistical analyses}

A detailed statistical analysis plan will be made available at the trial registry before analysis commences. Analysis of all outcomes will be on an intention-to-treat basis. Since complete twin allocations account for an estimated $20 \%$ of the study population and are independent risk factors for mortality [26], adjustment for twin correlation will be undertaken using linear mixed effects models for continuous data and generalised estimating equations for binary data.

\section{Comparability of participants in two arms}

Baseline characteristics will be presented by the allocation arm using descriptive statistics. Key indicators of standard hospital care received will be compared for both arms at baseline and during admission.

\section{Flow of participants}

The number and flow of subjects through screening, randomisation, allocation, follow-up and analysis will be documented, as per CONSORT 2010 guidelines [27], with reasons for exclusion, withdrawal and non-analysis being described (Fig. 5). Participants will be excluded from the final analysis if they have been permanently withdrawn.

\section{Primary and secondary outcome analysis}

The number of subjects with the primary outcome will be calculated for each arm and generalised estimating equations used to calculate risk ratios and the number needed to treat with confidence intervals. Analysis of secondary outcomes will be performed according to the type of data and using either number of subjects or person time as the denominator. Continuous variables will be compared between arms using random effects models, and categorical data with generalised estimating equations. Survival analysis of the time to death within first 28 days after birth will be performed using cox regression with frailty. In the event of multiple events for the same participant (e.g., infection), each episode will be considered an isolated event.

Missing data are expected to be few and will be addressed with a complete case analysis. Sub-group analyses for all outcomes will be performed according to birth weight categories $(<1200 \mathrm{~g} ; \geq 1200 \mathrm{~g})$ and multiple birth. Tests for effect modification by weight and multiple birth will be performed. The following will be calculated for both arms as indicators of adherence: mean chronological age at first KMC contact, mean time since admission at first KMC contact, daily dose of KMC (hours per study day) and average daily dose of in- 


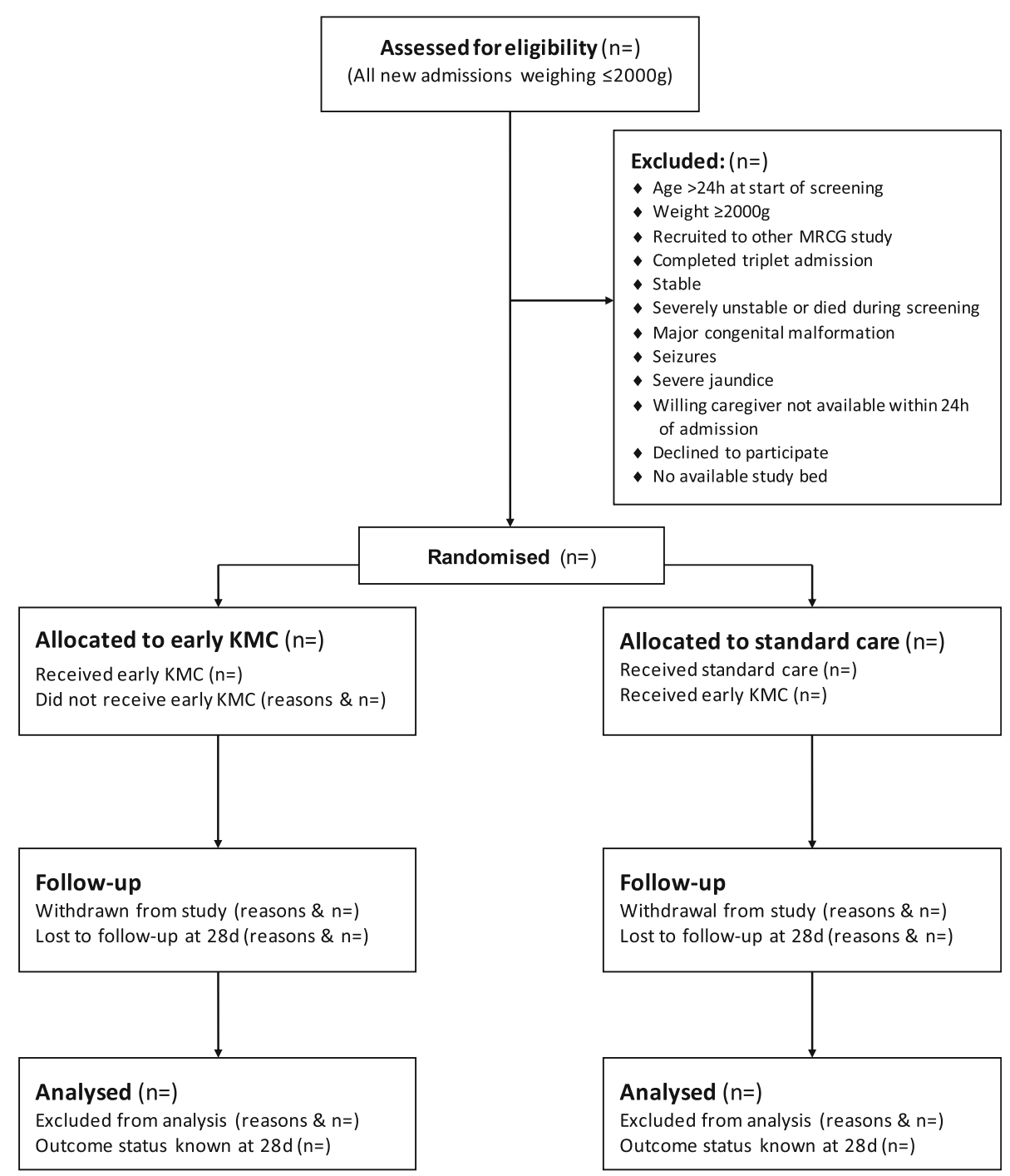

Fig. 5 Trial flow diagram, as per CONSORT guidelines 2010 [27]

patient KMC (per number of days admitted from enrolment). A sensitivity analysis of all outcomes will be performed according to average in-patient daily dose of KMC.

\section{Safety reporting and study monitoring against ICH-GCP standards}

Adverse events are any clinical event resulting in a change in management of the participant after enrolment and until age 28 days. Serious adverse events (SAE) are defined as death, life-threatening events (e.g., apnoea requiring bag-valve-mask ventilation, or severe instability), events carrying a risk of permanent or temporary disability (e.g., suspected meningitis), re-hospitalisation within 28 days of age and prolonged hospitalisation for $\geq 28$ days.

A local safety monitor, the sponsor and the trial monitors are informed of all SAEs within $24 \mathrm{~h}$ of the study team being aware with a detailed report sent within 2 working days for fatal and 5 days for a non-fatal SAE. All fatal SAEs are reported to the ethics committees monthly and within 7 days if related to the intervention. Non-fatal SAE's are communicated to the ethics committees annually or within 14 days if related to the intervention. A Data Safety Monitoring Board (DSMB) receives a bi-monthly safety report with bi-annual meetings to monitor recruitment, progress and safety. DSMB members include the clinical trialist/statistician (chair), a neonatologist experienced in a similar setting, a West African clinical trialist and an independent statistician. An un-blinded interim analysis will be conducted after randomisation of $50 \%$ of target sample size with prespecified stopping rules for efficacy, using the HaybittlePeto rule $[28,29]$ and will inform recommendations to the Trial Steering Committee (TSC), who will make the 
final decision on study continuation. Study procedures and documents are monitored for compliance to $\mathrm{ICH}-$ GCP standards by MRCG monitors every 3-6 months, with auditing determined by the sponsor.

\section{Discussion}

Evaluating the effect of continuous KMC before full stabilisation is a global research priority, stated by WHO [6] with the potential to contribute towards reducing the unacceptably high global neonatal mortality, enabling progress towards the neonatal mortality target SDG3.2 by 2030, as well as promoting a family-centred approach to newborn care. eKMC is one of the first trials to address this evidence gap and is expected to provide robust evidence in addition to novel mechanistic insights, particularly regarding infections, which are one of the major pathways to mortality for preterm neonates.

$\mathrm{KMC}$ reduces severe infections $(6.6 \%$ vs $13.1 \%, \mathrm{RR}=$ $0.5,95 \%$ CI $0.36-0.69)$ and nosocomial infections (4\% vs $11 \%, \mathrm{RR}=0.35$, 95\% CI $0.22-0.54)$ with intermittent or continuous $\mathrm{KMC}$ in stable neonates [6]. However, previous KMC trials have lacked clear case definitions for infection and a paucity of microbiologically confirmed data are available from resource-limited settings [6]. eKMC will contribute towards understanding the infection prevention effects of $\mathrm{KMC}$ by using a validated nosocomial risk score [24] microbiological testing and exploration of impact on carriage of antimicrobial resistant bacteria.

During eKMC trial piloting, we identified important challenges, which are outlined below with mitigating approaches:

Challenge 1 - Recruitment: The unavailability of caregivers willing to consent and provide the intervention within $24 \mathrm{~h}$ after birth is a major recruitment barrier due to high rates of maternal illness or post-caesarean section and absence of other family members at the hospital during the early admission period. Sensitisation activities with pregnant women and their families and health workers are undertaken at referral centres to encourage recruitment. A high proportion of either severe instability or death occurs before or during the screening process, reflecting the high proportion of out-born neonates and a vulnerable population. Access to sufficient study beds for the intervention was a limiting factor, and the number of study beds was increased from 2 to 4 during the piloting period to facilitate recruitment.

Challenge 2 - Non-blinded trial: KMC could not be blinded for the family or researchers. Selection and allocation bias are prevented through rigorous screening and randomisation procedures with objective stability markers, and transparent reporting of nonrecruitment will be performed. Treatment bias is minimised via a protocolised approach to standard care with prospective monitoring of adherence, comparable clinical monitoring and caregiver education for both arms.

Challenge 3 - Twins: Like much of West Africa, the twin birth rate in The Gambia is high at 16.7/1000 livebirths [30] with greater risk of premature delivery and neonatal death compared to singletons $[26,30]$. Evaluation of the intervention in twins is essential for generalisability of results and to target the most vulnerable neonates. Investigators anticipate that 30\% of participants will be twin gestation, with complete twin enrolment accounting for $20 \%$ of the study population. This may lead to differences in provision of the intervention in addition to independently impacting the trial outcomes. All efforts to adjust for multiple births will be made during analysis.

Challenge 4- Improvements to standard care leading to potential dilution of the intervention effect and risk of inadequate power: Alongside externally driven improvements to newborn care at the study site, eKMC implementation has resulted in major improvements to standard care for both trial and non-trial neonates. In collaboration with the hospital, the Gambian Government Ministry of Health and Social Welfare and UNICEF The Gambia, an eight-bed KMC unit was established, and continuous KMC was embedded in standard care in 2017. A protocolised approach to standard care of preterm neonates was also introduced at the site to reduce the risk of treatment bias. Although highly beneficial from an individual patient perspective, these improvements in care are expected to reduce both the power of detecting a difference in the primary outcome and may reduce differences between allocation arms, diluting the intervention effect. These changes to standard care will be explored in a linked process evaluation, based on the MRC guidance for evaluation of complex interventions [31] and using data collected before and after trial implementation. Activities will include a survival analysis of neonatal case fatality rates using published data from the study site [20] and prospective data collection for all admissions over the trial period, tracking of the changes made to standard newborn care, and KMC implementation progress monitoring [32].

If early KMC for pre-stabilised neonates is shown to be beneficial, we need to understand how to implement in a real-world setting. eKMC-generated implementation and safety data will be valuable, particularly when combined with similar trials, such as the multi-site OMWaNA trial 
in Uganda [33] and WHO-led multi-centre I-KMC trial [34]. We aim to align data definitions and maximise opportunities for pooled analyses with the OMWaNA trial.

The primary outcome results of the eKMC trial will contribute to the global evidence base for use of KMC before stabilisation in preterm neonates, with secondary outcome results and other analyses providing insights to how KMC is effective, particularly regarding infection prevention. The eKMC trial aims to inform one of the great divides between resource-limited and resource-rich settings and improve the chance for newborns everywhere to survive and thrive.

\section{Supplementary information}

Supplementary information accompanies this paper at https://doi.org/10. 1186/s13063-020-4149-y.

Additional file 1. SPIRIT 2013 Checklist: Recommended items to address in a clinical trial protocol and related documents.

Additional file 2. aSCRIP definition.

\section{Abbreviations}

AE: Adverse events; aPSBI: Adapted possible severe bacterial infection aSCRIP: Adapted stability of cardio-respiratory in preterm infants;

Cl: Confidence interval; CLSI: Clinical and Laboratory Standards Institute; CONS: Coagulase-negative staphylococcus; CPAP: Continuous positive airway pressure; CSF: Cerebral-spinal fluid; DSMB: Data safety monitoring board; EFSTH: Edward Francis Small Teaching Hospital; ESBL: Extended-spectrum beta lactamase; ISO: International Organisation for Standardisation; IV: Intravenous; KMC: Kangaroo mother care; LSHTM: London School of Hygiene \& Tropical Medicine; MRCG: Medical Research Council Unit The Gambia at London School of Hygiene \& Tropical Medicine; NMR: Neonatal mortality rate; PI: Principal investigator; RCT: Randomised controlled trial; RR: Risk ratio; SAE: Serious adverse event; SD: Standard deviation; SDGs: Sustainable development goals; TSC: Trial Steering Committee; VBA: Visual Basic Application; WHO: World Health Organisation

\section{Acknowledgements}

We thank the Members of the Trial Management Group at MRCG (Bunja Kebbeh, Alpha Jallow, Saffiatou Darboe, Mamadou Jallow, Buntung Ceesay, Yusupha Njie, and Binta Saidy), the Trial Steering Committee (Professor Enitan Carrol, Professor Elizabeth Mason, Professor Diana Elbourne, Dr. Baderinwa Abatan, Dr. Jane Crawley, and Professor Chinyere Ezeaka), Dr. Uduak Okomo (local safety monitor), Professor Elizabeth Allen, Dr. Melissa Morgan, Swetlana Kapoor, Onsagar Kapoor and Aidan Matthews.

\section{Trial status}

Recruitment began on 23 May 2018 and is expected to continue until June 2020. Protocol date and version: V4.0, 18 March 2019

Revision chronology:

V1.2, 14 February 2018: Original protocol approved.

V2.1, 7 July 2018, Amendment 1 (substantial, made in response to internal pilot phase and recommendations from TSC and DSMB) and including a reduction in the secondary outcomes, adjustment in the definition of $A E$ and SAEs to better reflect population of unstable neonates, randomisation blocks changed to not specify size of permuted blocks, strengthening of allocation procedures, window period of 5 days included for follow-up, blood and CSF samples transportation windows adjusted, SAE reporting period prolonged for non-fatal SAEs and DSMB reporting timelines changed to bi-monthly safety reports, stopping criteria expanded from 5 to 10 min in event of severe instability, and minor changes made to definitions of source documents and collection of data in electronic formats

V3.0, 4 Sept 2018, Amendment 2 (substantial, made in response to external changes to standard care at study site which impacted on stability definitions), including a change to the stability definition to include the use of bubble CPAP, a reduction in the intensity of the study assessments once participants become stable, removal of continuous temperature monitoring, removal of environmental temperature monitoring, and changes made in how the patient flow is recorded.

V4.0, 18 March 2019, Amendment 3 (non-substantial), including clarification of the definition of clinically suspected infection.

\section{Trial sponsor}

London School of Hygiene \& Tropical Medicine (LSHTM)

Sponsors reference: QA1078

Contact name: Mrs. Patricia Henley

Address: London School of Hygiene \& Tropical Medicine, London, UK Telephone: +4420 79272626

Email: patricia.henley@lshtm.ac.uk

The study sponsor and funder had no role in the study design, data collection, study management or planned analysis. They had no input into the writing of the report or decision to submit for publication and do not have any authority over any of the above activities. Participants will receive compensation for any harm suffered as a result of the trial as per the sponsor's standard indemnity.

\section{Composition, roles and responsibilities of the trial team} Trial Steering Committee:

Professor Enitan Carrol, University of Liverpool (Chair); Professor Elizabeth Mason; Professor Chinyere Ezeaka; Dr. Jane Crawley; Dr. Baderinwa Abatan; Professor Diana Elbourne; Professor Simon Cousens; Professor Joy Lawn; Dr. Anna Roca; Dr. Helen Brotherton

Role: Overview of study design \& conduct; receipt of DSMB reports and action as appropriate; publication \& dissemination of results

Trial Management group:

Dr. Helen Brotherton, Dr. Anna Roca, Dr. Abdou Gai, Mr Bunja Kebbeh, Mr Alpha Jallow, Mr Yusupha Njie, Miss Binta Saidy, Mrs Saffiatou Darboe Role: Responsible for organisation and day-to-day conduct of the study; maintenance of data-entry, cleaning and verification system; laboratory management and financial administration of the study MRCG Trial Monitors team:

Vivat Thomas Njie, Gibbi Sey, Fatou Joof, Maxine Heffner

Role: Monitoring progress of the study against ICH-GCP standards eKMC Data Safety \& Monitoring Board:

Professor Pollyanna Hardy, University of Birmingham (chair); Dr. Christabel Enweronu-laryea; Dr. Martin Ota; Dr. Anne Segonds-Pichon. Terms of reference for the DSMB are available on request from the eKMC PI (HB, corresponding author).

\section{Authors' contributions}

$\mathrm{HB}$ and JEL conceived of the research question, obtained the funding from Wellcome Trust and designed the protocol with substantial input from SMAZ, SC, ACS, AR and CJT. The study protocol was implemented by HB, AR, $A G$ and $A L S$. HB wrote the first version of the manuscript. All authors read and approved the final version of the manuscript.

\section{Funding}

This work is funded by The Wellcome Trust (Ref. 200116/Z/15/Z) as a Research Training Fellowship to HB. The funders played no role in the trial design or conduct and will not contribute to the analysis or interpretation of the data. They did not contribute towards the writing of this manuscript.

\section{Availability of data and materials}

The final trial dataset will be available on requests made to the $\mathrm{PI}$ or institutional delegate. The results of this study will be published in an open access format in a peer-reviewed biomedical journal, in addition to the PI's doctoral thesis. The results will be disseminated at relevant international scientific forums and communicated to the World Health Organisation. The Gambian Government Ministry of Health and Social Welfare and other relevant local stakeholders and participant families will be directly informed of the study results.

Ethics approval and consent to participate

Ethical approval was obtained for the study protocol and informed consent documents from LSHTM Intervention Ethics Committee (Ref. 14545) and Gambian Government/MRCG Joint Ethics Committee (Ref. 1591). Any 
substantial changes to the study protocol which may impact on the study conduct or patient safety will be submitted to the ethics committees, and after approval, the sponsor, trial registry, TSC and DSMB will be notified. Details of the consent procedures are outlined in the main manuscript.

\section{Consent for publication}

Consent for obtaining the photograph (Fig. 3) and for publication was obtained from the caregiver featured in the photograph, using the MRCG at LSHTM photographic consent form.

\section{Competing interests}

The authors declare that they have no competing interests. The funder (Wellcome Trust) played no role in the design of the trial or writing of the manuscript.

\section{Author details}

${ }^{\top}$ Faculty of Epidemiology and Population Health, and MARCH Centre, London School of Hygiene \& Tropical Medicine (LSHTM), Keppel Street, London, UK. ${ }^{2}$ MRC Unit The Gambia at LSHTM, Atlantic Road, Fajara, The Gambia. ${ }^{3}$ Department of Medical Paediatrics, Royal Hospital for Sick Children, Edinburgh, UK. ${ }^{4}$ MRC/UVRI \& LSHTM Uganda Research Unit, Plot 51-59 Nakiwogo Road, Entebbe, Uganda. ${ }^{5}$ Neonatal Medicine, University College London Hospitals NHS Trust, 235 Euston Rd, London, UK. ${ }^{6}$ Ministry of Health and Social Welfare, Gambia Government, Banjul, The Gambia. ${ }^{7}$ Education Department, Liverpool School of Tropical Medicine, Pembroke Place, Liverpool, UK

Received: 12 September 2019 Accepted: 6 February 2020 Published online: 06 March 2020

\section{References}

1. Chawanpaiboon S, Vogel JP, Moller AB, Lumbiganon P, Petzold M, Hogan D Landoulsi S, Jampathong N, Kongwattanakul K, Laopaiboon M, Lewis C, Rattanakanokchai S, Teng DN, Thinkhamrop J, Watananirun K, Zhang J, Zhou W, Gülmezoglu AM. Global, regional, and national estimates of levels of preterm birth in 2014: a systematic review and modelling analysis. Lancet Glob Health. 2019;7(1):e37-46.

2. Liu L, Oza S, Hogan D, Chu Y, Perin J, Zhu J, Lawn JE, Cousens S, Mathers C, Black RE. Global, regional, and national causes of child mortality in 2000-15, an updated systematic analysis with implications for the sustainable development goals. Lancet. 2016;388(10063):3027-35.

3. Sankar MJ, Natarajan CK, Das RR, Agarwal R, Chandrasekaran A, Paul VK. When do newborns die? A systematic review of timing of overall and cause-specific neonatal deaths in developing countries. J Perinatol. 2016;36: S1-S11.

4. Lawn JE, Blencowe H, Oza S, You D, Lee AC, Waiswa P, Lalli M, Bhutta Z, Barros AJ, Christian P, Mathers C, Cousens SN, Lancet Every Newborn Study Group. Every newborn: progress, priorities, and potential beyond survival. Lancet. 2014;384(9938):189-205.

5. World Health Organisation. WHO recommendations on interventions to improve preterm birth outcomes. Geneva: World Health Organisation; 2015.

6. Conde-Agudelo A, Díaz-Rossello JL. Kangaroo mother care to reduce morbidity and mortality in low birthweight infants. Cochrane Database Syst Rev. 2016;(8):CD002771. https://doi.org/10.1002/14651858.CD002771.pub4.

7. Charpak N, Ruiz-Peláez JG, de Fiqueroa CZ, Charpak Y. Kangaroo mother versus traditional care for newborn infants $</=2000$ grams: a randomized, controlled trial. Pediatrics. 1997;100(4):682-8.

8. Lawn JE, Mwansa-Kambafwile J, Horta BL, Barros FC, Cousens S. Kangaroo mother care to prevent neonatal deaths due to preterm birth complications. Int J Epidemiol. 2010;39:1144-54.

9. Boundy EO, Dastjerdi R, Spiegelman D, Fawzi WW, Missmer SA, Lieberman E, Kajeepeta S, Wall S, Chan GJ. Kangaroo mother care and neonatal outcomes: a meta-analysis. Paediatrics. 2016;137(1). https://doi.org/10.1542/ peds.2015-2238.

10. Worku B, Kassie A. Kangaroo mother care: a randomized controlled trial on effectiveness of early kangaroo mother care for the low birthweight infants in Addis Ababa, Ethiopia. J Trop Pediatr. 2005;51(2):93-7.

11. Lorenz L, Dawson JA, Jones H, Jacobs SE, Cheong JL, Donath SM, Davis PG, Kamlin COF. Skin-to-skin care in preterm infants receiving respiratory support does not lead to physiological instability. Arch Dis Child Fetal Neonatal Ed. 2017;102(4):F339-44.
12. Vittner D, McGrath J, Robinson J, Lawhon G, Cusson R, Eisenfeld L, Walsh S,

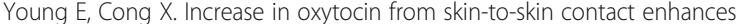
development of parent-infant relationship. Biol Res Nurs. 2017;20(1):54-62.

13. Mörelius E, Örtenstrand A, Theodorsson E, Frostell A. A randomised trial of continuous skin-to-skin contact after preterm birth and the effects on salivary cortisol, parental stress, depression, and breastfeeding. Early Hum Dev. 2015;91(1):63-70.

14. Chi Luong K, Long Nguyen T, Huynh Thi DH, Carrara HP, Bergman NJ. Newly born low birthweight infants stabilise better in skin-to-skin contact than when separated from their mothers: a randomised controlled trial. Acta Paediatr. 2016;105(4):381-90.

15. Hendricks-Muñoz KD, Xu J, Parikh HI, Xu P, Fettweis JM, Kim Y, Louie M, Buck GA, Thacker LR, Sheth NU. Skin-to-skin care and the development of the preterm infant oral microbiome. Am J Perinatol. 2015;32(13):1205-1.

16. Chan AW, Tetzlaff JM, Gøtzsche PC, Altman DG, Mann H, Berlin JA, Dickersin K, Hróbjartsson A, Schulz KF, Parulekar WR, Krleza-Jeric K, Laupacis A, Moher D. SPIRIT 2013 explanation and elaboration: guidance for protocols of clinical trials. BMJ. 2013;346:e7586

17. United Nations Development Program. Human development indices and indicators statistical update. New York; 2018. http://hdr.undp.org/en/2019update. Accessed 9 Aug 2019.

18. United Nations Inter-Agency Group for Child Mortality Estimation. Levels \& trends in child mortality: report 2019. New York. https://www.unicef.org/media/60561/ file/UN-IGME-child-mortality-report-2019.pdf. Accessed 31 Jan 2020.

19. Blencowe H, Cousens S, Oestergaard M, Chou D, Moller AB, Narwal R, Adler A, Garcia CV, Rohde S, Say L, Lawn JE. National, regional and worldwide estimates of preterm birth rates in the year 2010 with time trends since 1990 for selected countries: a systematic analysis and implications. Lancet. 2012;379(9832):2162-7.

20. Okomo UA, Dibbasey T, Kassama K, Lawn JE, Zaman SM, Kampmann B, Howie SR, Bojang K. Neonatal admissions, quality of care and outcome: 4 years of inpatient audit data from The Gambia's teaching hospital. Paediatr Int Child Health. 2015;35(3):252-64.

21. Moxon SG, Lawn JE, Dickson KE, Simen-Kapeu A, Gupta G, Deorari A, Singhal N, New K, Kenner C, Bhutani V, Kumar R, Molyneux E, Blencowe H. Inpatient care of small and sick newborns: a multi-country analysis of health system bottlenecks and potential solutions. BMC Pregnancy Childbirth. 2015;15(Suppl 2):S7.

22. Fischer CB, Sontheimer D, Scheffer F, Bauer J, Linderkamp O. Cardiorespiratory stability of premature boys and girls during kangaroo care. Early Hum Dev. 1998;52(2):145-53.

23. Young Infants Clinical Signs Study Group. Clinical signs that predict severe illness in children under age 2 months: a multicentre study. Lancet. 2008; 371(9607):135-42.

24. Rosenberg RE, Ahmed AS, Saha SK, Chowdhury MA, Ahmed S, Law PA, Black RE, Santosham M, Darmstadt GL. Nosocomial sepsis risk score for preterm infants in low-resource settings. J Trop Pediatr. 2010;56(2):82-9.

25. Ballard JL, Khoury JC, Wedig K, Wang L, Eilers-Walsman BL, Lipp R. The New Ballard Score, expanded to include extremely premature infants. J Pediatr. 1991;119:417-23.

26. Bjerregaard-Andersen M, Lund N, Jepsen FS, Camala L, Gomes MA, Christensen K, Christiansen L, Jensen DM, Aaby P, Beck-Nielsen H, Benn CS, Sodemann M. A prospective study of twinning and perinatal mortality in urban Guinea-Bissau. BMC Pregnancy Childbirth. 2012;12:140.

27. Schulz KF, Altman DG, Moher D. CONSORT 2010 statement: updated guidelines for reporting parallel group randomised trials. Int J Surg. 2011;9(8):672-7.

28. Haybittle JL. Repeated assessment of results in clinical trials of cancer treatment. Br J Radiol. 1971;44(526):793-7.

29. Peto R, Pike MC, Armitage P, Breslow NE, Cox DR, Howard SV, Mantel N, McPherson K, Peto J, Smith PG. Design and analysis of randomized clinical trials requiring prolonged observation of each patient. II. Analysis and examples. Br J Cancer. 1977:35(1):1-39.

30. Reiko M, Jasseh M, Mackenzie GA, Bottomley C, Jahar M, Greenwood B, D'Alessandro U, Roca A. The large contribution of twins to neonatal and post-neonatal mortality in The Gambia, a 5-year prospective study. BMC Pediatr. 2016:16:39.

31. Moore GF, Audrey S, Barker M, Bond L, Bonell C, Hardeman W, Moore L, O'Cathain A, Tinati T, Wight D, Baird J. Process evaluation of complex interventions: Medical Research Council guidance. BMJ. 2015;350:h1258.

32. Bergh AM, Manu R, Davy K, van Rooyen E, Asare GQ, Williams JK, Dedzo M, Twumasi A, Nang-Beifubah A. Translating research findings into practice-the 
implementation of kangaroo mother care in Ghana. Implement Sci. 2012; 75.

33. Morgan M. Kangaroo mother care before stabilisation amongst low birth weight neonates in Africa. (OMWaNA). ClinicalTrials.gov. 2016. https:// clinicaltrials.gov/ct2/show/NCT02811432?cond=NCT03555981. Accessed 29 Aug 2019.

34. Rajiv Bahl. Effect of immediate kangaroo mother care on neonatal mortality for mothers and babies (I-KMC). ANZCTR. 2018. https://www.anzctr.org.au/ Trial/Registration/TrialReview.aspx?id=375825. Accessed 8 Sept 2019.

\section{Publisher's Note}

Springer Nature remains neutral with regard to jurisdictional claims in published maps and institutional affiliations.

Ready to submit your research? Choose BMC and benefit from:

- fast, convenient online submission

- thorough peer review by experienced researchers in your field

- rapid publication on acceptance

- support for research data, including large and complex data types

- gold Open Access which fosters wider collaboration and increased citations

- maximum visibility for your research: over $100 \mathrm{M}$ website views per year

At $\mathrm{BMC}$, research is always in progress.

Learn more biomedcentral.com/submissions 